\title{
Effect of Naso-respiratory Obstruction with Mouth Breathing on Dentofacial and Craniofacial Development
}

\author{
Dr Swati Saraswata Acharya, Dr Lipika Mali,, Dr Abhik Sinha, ${ }^{3}$ Dr Smruti Bhusan Nanda \\ ${ }^{\prime}$ Assoc Prof, 2.3 PG Student, 4Prof, Dept of Orthodontics, Institute of Dental Sciences, \\ SOA University, Bhubaneswar, India
}

Correspondence: Dr. Swati Acharya; Email: swati.acharya.tmdc@gmail.com

\section{ABSTRACT}

Objective: To evaluate the effects of mouth breathing on craniofacial and dentofacial development during childhood in comparison to nasal breathing in malocclusion patients.

Materials \& Method: A retrospective study done at SOA University. Cephalometric parameters and clinical variables of 90 pediatric patients who had undergone orthodontic treatment were reviewed. Study group included 40 pediatric patients who suffered from signs and symptoms of nasal obstruction, and control group included 50 patients who were normal nasal breathers. Dental and craniofacial parameters were compared between nasal breathers and mouth breathers using clinical and cephalometric records.

Result: The mouth breathers had backward and downward rotation of mandible with increased overjet, increased mandibular plane angle, higher palatal plane, and constriction of upper and lower arches at the level of cuspids and first molars when compared with nasal breathers group. The prevalence of posterior cross bite was observed greater in mouth breathers group $(40 \%)$ than the nose breathers $(20 \%)(p=0.006)$. Abnormal lip-to-tongue anterior oral seal was seen more in the mouth breathers group (55\%) than in nose breathers group (25\%) $(p=0.05)$.

Conclusion: Naso-respiratory obstruction with mouth breathing during growth periods in children has a greater tendency for clockwise rotation of growing mandible, with an irregular increase in anterior lower vertical face height and decreased posterior facial height.

Keywords: anterior oral seal, dentofacial growth, mouth breathing, malocclusion, nasal obstruction

\section{INTRODUCTION}

The relationship between nasal obstruction and craniofacial growth is highly debatable in literature.' Much of the disagreement relates to lack of sophistication in quantifying nasal versus oral respiration and deficiency of longitudinal data. According to Moss's theory of functional matrix, nasal breathing allows growth and development of dentofacial complex. ${ }^{2}$ This theory is entirely based on the principle that normal nasal respiratory activity has an impact on the development of craniofacial structures, favoring harmonious growth and development by interacting with mastication and swallowing along with other components of head and neck region. ${ }^{3,4}$ Chronic nasal obstruction leads to mouth breathing, resulting in an anterior or lower position of the tongue, incompetent lips, lowered position of the mandible, and decreased orofacial muscle tonicity to compensate for decreased nasal airflow and facilitate respiration..$^{5-8}$ Hence, there is disharmony in growth and development of orofacial structures, narrowing of maxilla, underdevelopment of mandible, alterations in the position of head in relation to the neck, protrusion of maxillary incisors, and distal position of mandible in relation to maxilla. ${ }^{9}$

Mouth breathing has a multifactorial etiology which varies from anatomical obstruction like palatine and pharyngeal tonsil hypertrophy, nasal polyp, septal deviation, allergic rhinitis, nasal turbinate hypertrophy and deleterious oral habits that might deform the dental arch and alter facial harmony depending on intensity, duration and frequency of such habits. ${ }^{10,11}$ The most common cause of mouth breathing is nasal obstruction, specifically adenoid hypertrophy in pediatric population. ${ }^{12}$ In children, the phenomenon of mouth breathing is important as it adversely influences growth and development. The children with chronic mouth breathing might develop morphological disorders during the growth phase resulting in unfavorable development of craniofacial and dentofacial complex. However, relationship between 
mouth breathing and dentofacial development is controversial as some authors do not associate nasal obstruction as a significant factor causing abnormal dentofacial and craniofacial development. ${ }^{13-15}$

The purpose of this study was to evaluate the effects of mouth breathing during childhood on craniofacial and dentofacial development in comparison to nasal breathing in malocclusion patients treated in orthodontic department.

\section{MATERIALS AND METHOD}

A retrospective review of 90 pediatric patients who had undergone orthodontic treatment at the Department of Orthodontics from 2012 to 2016 was performed. Cephalometric and clinical parameters of those patients were reviewed. Study group included 40 pediatric patients who suffered from the signs and symptoms of nasal obstruction as a result of mouth breathing (Table 1). The control group included 50 patients who were normal nasal breathers. Patients in both groups were orthodontically treated. Mode of breathing was illustrated according to history and clinical examination which included anterior rhinoscopy, lateral nasopharyngeal x-ray, flexible nasopharyngoscopy, and then confirmed by questionnaires answered by patients' parents (Table 2). ${ }^{16}$ The two groups did not have any past history of nasorespiratory surgery. The examined parameters during orthodontic evaluation included demographic data, respiratory status of patient, comprehensive oral examination, cephalometric analysis, and study models. The study models were evaluated and measured for arch form, symmetry, position of tooth,

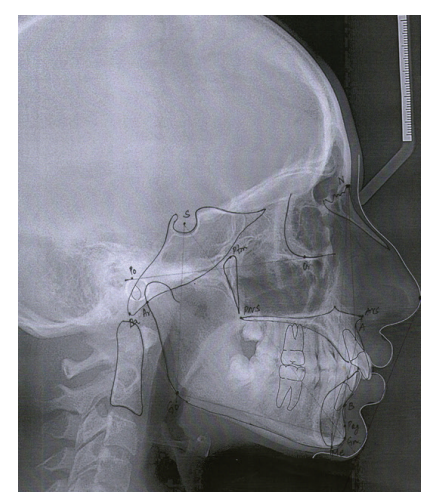

Figure 1. Cephalometric landmarks

occlusion, and other parameters liable to undergo changes because of mouth breathing.

Cephalometric analysis was made on standardized lateral head plates to compare parameters that might be influenced by different modes of breathing in two groups. The performed cephalometric analysis was used to define planes and angles representing deviations from accepted norms in affected patients. Cephalometric points marked in cephalograms taken in Frankfort Horizontal position are described in Figure 1. After locating landmarks of anatomical skeletal points, angular and linear cephalometric measurements were obtained. The data were collected in a table and subjected to statistical analysis for determination of differences.

The data were evaluated using descriptive statistical methods. Differences between groups were analyzed by using $v 2$ test for categorical variables. Statistical tests were performed using SPSS version 12.0 software. Statistical significance was accepted at a level $p<0.05$.

Table 1. Demographic characteristics of samples

\begin{tabular}{|c|c|c|c|}
\hline \multicolumn{2}{|c|}{ Parameter } & Mouth Breathers, $n=40(\%)$ & Nose Breathers, $n=50(\%)$ \\
\hline \multirow{2}{*}{ Gender } & Female & $20(19.1 \%)$ & $30(50.4 \%)$ \\
\hline & Male & $20(29.9 \%)$ & $20(40.6 \%)$ \\
\hline \multirow{2}{*}{ Age (years) } & Mean $\pm S D$ & $12.49 \pm 1.94$ & $12.55 \pm 2.11$ \\
\hline & Range & $10-14$ & $10-14$ \\
\hline
\end{tabular}

Table 2. Breathing Mode according to Questionnaire

\begin{tabular}{|l|c|c|}
\hline \multicolumn{1}{|c|}{ Parameter } & Mouth Breathers $(\mathbf{n = 4 0 )}$ & Nose Breathers $(\mathbf{n = 5 0})$ \\
\hline Snoring & 31 & 0 \\
\hline Sleep apnea & 5 & 0 \\
\hline Allergic rhinitis & 7 & 0 \\
\hline Closed rhinolalia & 6 & 0 \\
\hline Mouth open at rest & 35 & 0 \\
\hline Drooling on pillar & 35 & 0 \\
\hline Recurrent otitis media & 4 & 0 \\
\hline Chronic middle ear effusion & 5 & 0 \\
\hline
\end{tabular}




\section{RESULT}

Dental and craniofacial parameters of mouth breathers and nose breathers are shown in Table 3. In horizontal, vertical, and lateral dimensions there were significant differences between mouth breathers and nose breathers groups. The horizontal dimension showed an increased overjet in mouth breathing group with backward and downward rotation of the mandible, which was represented by the lower lip to E-line distance. The vertical dimension evaluated a significant increase in mandibular plane angle (GoGn to SN), an increase in the y-axis angle and a higher palatal plane. The lateral dimension of arches showed narrowing of both upper and lower arches at the level of canines and first molars in mouth breathers group. However, other cephalometric landmarks, like SNA angle, overbite, and ANB angle, did not show any significant differences between mouth breathers and control group.
The distribution of Angle's classification and anterior and posterior crossbite malocclusions in mouth breathers and nose breathers is shown in Table 4. In the study population most of them presented with Class II malocclusion (66\%). No significant differences in malocclusion classification was found between mouth breathers and the nose breathers $(p=0.49)$. However, a tendency toward an increased rate of Class ॥ malocclusion was found among mouth breathers (70\%). In mouth breather group, Class II was three times more common than Class I. In fact, when comparing prevalence of posterior crossbite between the two groups, posterior crossbite was significantly more frequent in the mouth breathers group (40\%) than nose breathers $(20 \%)(p=0.006)$.

The relationship between anterior oral seal (AOS) and mode of breathing is shown in Table 5. Most of the nose breathers had normal lip-to-lip AOS during swallowing (70\%). However, an abnormal lip-to-tongue AOS was more frequent in the mouth breathers group (55\%) than nose breathers group (25\%) ( $p=0.05)$.

Table 3. Dental and craniofacial parameters of mouth breathers and nose breathers

\begin{tabular}{|c|c|c|c|c|}
\hline Dimension & Parameter & $\begin{array}{c}\text { Mouth Breathers, } n=40(\%) \\
\text { (Mean } \pm S D)\end{array}$ & $\begin{array}{c}\text { Nose Breathers, } n=50(\%) \\
\text { (Mean } \pm \text { SD) }\end{array}$ & $p$-Value \\
\hline \multirow{5}{*}{ Horizontal } & Overjet, mm & $5.95 \pm 3.31$ & $4.47 \pm 2.53$ & $0.01^{*}$ \\
\hline & SNB angle & $74.84 \pm 3.94$ & $75.96 \pm 3.61$ & $0.002^{*}$ \\
\hline & SNA angle & $78.61 \pm 6.61$ & $81.35 \pm 7.88$ & 0.2 \\
\hline & ANB angle & $4.43 \pm 2.81$ & $3.39 \pm 9.00$ & 0.416 \\
\hline & Lower lip to E-line, $\mathrm{mm}$ & $1.43 \pm 2.77$ & $0.06 \pm 2.84$ & $0.01 *$ \\
\hline \multirow{6}{*}{ Vertical } & ANS-Me, mm & $66.66 \pm 6.40$ & $65.07 \pm 6.00$ & 0.038 \\
\hline & Go-GN to SN angle & $35.85 \pm 5.80$ & $33.76 \pm 4.53$ & $0.002^{*}$ \\
\hline & Y-axis angle & $70.45 \pm 4.20$ & $65.27 \pm 4.85$ & $0.002 *$ \\
\hline & Overbite & $2.85 \pm 2.34$ & $3.10 \pm 1.98$ & 0.521 \\
\hline & S-Go to N-Me, ratio in \% & $60.47 \pm 5.23$ & $64.66 \pm 2.41$ & $0.025^{*}$ \\
\hline & Palatal height, $\mathrm{mm}$ & $25.14 \pm 1.82$ & $22.89 \pm 2.21$ & $0.02^{*}$ \\
\hline \multirow{4}{*}{ Lateral } & Upper 3-3 width & $27.70 \pm 1.89$ & $30.78 \pm 2.09$ & $0.02^{*}$ \\
\hline & Upper 6-6 width & $31.21 \pm 2.06$ & $35.64 \pm 2.68$ & $0.02 *$ \\
\hline & Lower 3-3 width & $25.94 \pm 1.95$ & $28.30 \pm 1.96$ & $0.02 *$ \\
\hline & Lower 6-6 width & $31.62 \pm 2.00$ & $32.61 \pm 2.63$ & $0.02 *$ \\
\hline
\end{tabular}

*Statistically significant at $p<0.05$

Table 4. Prevalence of Angle's classification and posterior crossbite malocclusions in mouth breathers and nose breathers

\begin{tabular}{|c|c|c|c|c|}
\hline Dimension & Parameter & Mouth Breathers, $n=40$ (\%) & Nose Breathers, $n=50(\%)$ & $p$-Value \\
\hline \multirow{3}{*}{$\begin{array}{c}\text { Horizontal } \\
\text { (Angle's classification) }\end{array}$} & Class I & $10(22)$ & $21(31)$ & \multirow{3}{*}{0.48} \\
\hline & Class II & $27(73)$ & $25(62)$ & \\
\hline & Class III & $3(5)$ & $4(7)$ & \\
\hline Lateral & Posterior crossbite & $28(39)$ & $16(26)$ & $0.006^{*}$ \\
\hline
\end{tabular}


Table 5. Association between anterior oral seal and mode of breathing

\begin{tabular}{|l|c|c|c|}
\hline \multicolumn{1}{|c|}{ Anterior Oral Sealing (AOS) } & Mouth Breathers, $\mathbf{n = 4 0 ( \% )}$ & Nose Breathers, $\mathbf{n = 5 0}(\mathbf{\%})$ & $\mathbf{p}$-Value \\
\hline Normal AOS (lip to lip) & $15(45)$ & $30(70)$ & $0.05^{*}$ \\
\hline Abnormal AOS (lip to tongue) & $25(39)$ & $20(30)$ & \\
\hline
\end{tabular}

*Significant at $\mathrm{p} \leq 0.05$

\section{DISCUSSION}

The concepts that nasal obstruction and mouth breathing adversely affects the craniofacial and dentofacial development still ontinues to be controversial, and this is at least in part due to criterion used to define mouth breathing, which is often subjective.

In the present study, a significant difference was found between mouth breathers and control groups in horizontal, vertical, and lateral dimensions. In the horizontal dimension, mandible was located in a posterior position in relation to maxilla in mouth breather patients, which was demonstrated by an increased overjet, SNB angle, and distance between lower lip to E-line. These findings support previous evidence that retrognathic mandible and posterior inclination of mandibular plane in mouth breathing children are higher than the nasal breathing children. ${ }^{17-19}$

In the vertical dimension, posterior and anterior facial height ratio (S-Go to N-Me ratio) was lower in mouth breathing children, indicating proportionally lower posterior facial height than anterior facial height in these patients. This result confirms evidence that mouth breathing children present clockwise rotation of mandible (downward and posterior rotation) stimulating increased vertical growth of anterior portion of the face relative to posterior portion of the face. ${ }^{5}$ In the lateral dimension, there was narrowing of the upper and lower dental arches in mouth breathers group compared to control group.

The findings in three dimensions support previous theories that the mouth breathers have higher tendency for abnormal skeletal and dental growth in comparison to normal growth parameters. In Angle's classification we found an increased tendency towards class II in mouth breathers group, which indicates posterior position of mandible. Our finding was without any significant difference between both groups. The cause for this finding could be due to study population that has known malocclusion. However, prevalence of class II malocclusion in the mouth breathers (73\%) was higher than prevalence of class II malocclusion in Israeli population (28.1\%). ${ }^{20}$ However, in lateral dimension, a significant difference was found in the prevalence of posterior cross bite. This finding might be explained by the lower position of tongue followed by downward rotation of the mandible to enhance oral breathing exclusively. Elongation of buccinator muscles might create inward pressure on upper dental arch. When determining AOS we found a significant difference between both groups. The mouth breathers group showed increased rate of abnormal AOS (55\%) compared to the control group (25\%), which indicates inability to bring upper and lower lip together to create normal OS. The unique feature of our study in comparison to the previous studies is that orthodontic malocclusion was present in both groups, and the difference between them was mode of breathing.

Normal craniofacial and dentofacial development depends on various other factors. Genetic factors influence constitution of facial and occlusal pattern of an individual, and stomatognathic apparatus function plays an important role. 13,21,22 The craniofacial and dentofacial abnormalities that have been linked with nasal obstruction are usually featured with an elongated face, open-mouth posture, protrusive and proclined maxillary incisors, constricted maxillary arch, high-arched palate, and Angle's Class II malocclusion. The term 'adenoid face' encompasses most of these features. ${ }^{22-24}$

Upper airway obstruction induces a change in balance between forces and the pressure exerted by various musculature such as muscles of tongue, orbicularis oris, and buccinators, generating morphological changes in dental and craniofacial parameters. ${ }^{25,26}$ Some authors described a physiological mechanism that causes these changes in neuromuscular system as a result of upper respiratory airway obstruction. ${ }^{27}$ The neuromuscular changes encourage alteration in bony and soft tissues of dental and craniofacial structures (Figure 2). 
Nasal Obstruction

Air intake increase by means of oral breathing

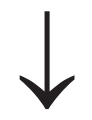

Change in functioning of the neuromuscular system of the craniofacial muscles

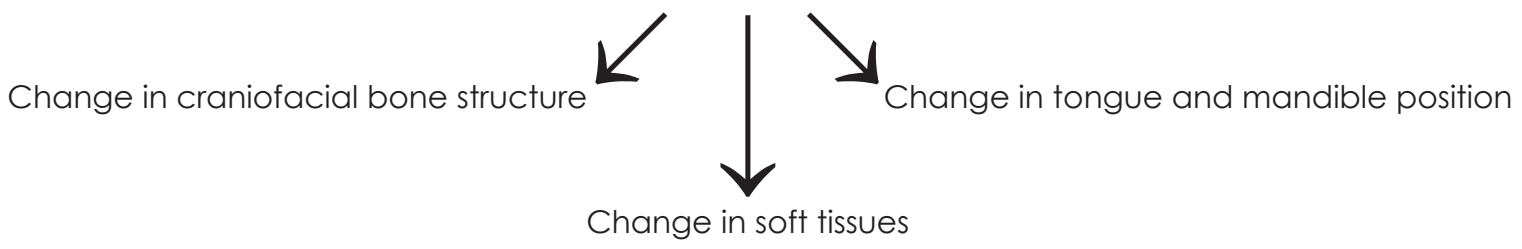

Figure 2. Relationship between naso-respiratory obstruction and craniofacial growth

Previous data and results from our study show that longstanding nasal obstruction which causes mouth breathing correlated with a negative impact on dentofacial and craniofacial development. In addition, mouth breathing has a similar effect on mandibular growth irrespective of its etiology. ${ }^{28-30}$

\section{CONCLUSION}

Nasorespiratory obstruction along with mouth breathing during critical growth periods in children has a higher tendency for clockwise rotation of growing mandible, with a disproportionate increase in the anterior lower vertical face height and decreased posterior facial height. Such increase in the anterior lower vertical face height is often associated with open bite and retrognathia Otolaryngologists, pediatricians, and orthodontists must take note of the chronic mouth breathing in children, since any delay in diagnosis and treatment might cause abnormal dentofacial and craniofacial development. They also have a significant role in the diagnosis and management of orthodontic patients since the signs and symptoms might be recognizable in the dental practice. 


\section{REFERENCES}

1. Warren DW, Spalding PM. Dentofacial morphology and breathing: a century of controversy. In: Melsen, B, editor. Current controversies in orthodontics. Chicago: Quintessence Pub Co; 1991. p. 45-76.

2. Moss ML. The functional matrix: functional cranial components. In: Kraus B, Riedel R, editors. Vistas in orthodontics. Philadelphia:Lea \& Febiger; 1962. p. 85-90.

3. Peltomaki T. The effect of mode of breathing on craniofacial growth- revisited. Eur J Orthod 2007;29:426-9.

4. Linder-Aronson S. Respiratory function in relation to facial morphology and the dentition. Angle Orthod. 1979;6:59-71.

5. Drake AF, Keall HJ, Vig PS, Krause CJ. Clinical nasal obstruction and objective respiratory mode determination. Ann Otol Rhinol Laryngol. 1988;97:397-402.

6. Kluemper GT, Vig PS, Vig KWL. Nasorespiratory characteristics and craniofacial morphology. Eur J Orthod. 1995;491-5.

7. Vig PS, Kowalski CJ. Interrelations between respiratory mode, nasal resistance, flowrate and cross-sectional area. J Dent Res. $1991 ; 72: 342$.

8. Riolo ML, Moyers RE, McNamara JA, Hunter WS. An atlas of craniofacial growth. Monograph \#2 Craniofacial Growth Series. Ann Arbor: University of Michigan; 1979; p. 25-200.

9. Fields HW, Warren DW, Black K, Phillips CL. Relationship between vertical dentofacial morphology and respiration in adolescents. Am J Orthod Dentofac Orthop. 1991:99:147-54.

10. Valera FCP, Travitzki LVV, Mattar SEM, Matsumoto MAN, Elias AM, Anselo-Lima WT. Muscular, functional, and orthodontic changes in preschool children with enlarged adenoids and tonsils. Int J Pediatr Otorhinolaryngo.I 2003;67:761-770.

11. Andrade LP, Majolo MS. A infliencia da respiracao bucal no crescimento facial. Rev Goiana Orthod. 2000;5:34-45.

12. Rubin RM. Mode of respiration and facial growth. Am J Orthod. 1980;78:504-10.

13. Tourne LPM, Scheweiger J. Immediate postural responses to total nasal obstruction. Am J Dentofac Orthop. 1996; 1 10:606-11.

14. Kawashima S, Peltomaki T, Sakata H, Mori K, Happonen RP, Ronning O. Craniofacial morphology in preschool children with sleep-related breathing disorder and hypertrophy of tonsils. Acta Paediatr. 2002;91:71-7.

15. Nowak AJ, Warren JJ. Infant oral health and oral habits. Pediatr Clin North Am. 2000;47:1043-66,vi.

16. Li AM, Cheung A, Chan D. Validation of a questionnaire instrument for prediction of obstructive sleep apnea in Hong Kong Chinese children. Pediatr Pulmonol. 2006;41:1153-60.

17. Sousa JB, Anselmo-Lima WT, Valera FC, Gallego AJ, Matsumoto MA. Cephalometric assessment of the mandibular growth pattern in mouth-breathing children. Int J Pediatr Otorhinolaryngol. 2005;69:311-7.

18. Prince ME, Nasser JG, Pass BB. Effect of upper-airway passages on craniofacial growth in an animal model: a pilot study. J Otolaryngol. 1997:26:306-15.

19. Aboudara C, Nielsen I, Huang JC, Maki K, Miller AJ, Hatcher D. Comparison of airway space with conventional lateral headfilms and 3-dimensional reconstruction from cone-beam computed tomography. Am J Orthod Dentofac Orthop. 2009;135:468-79.

20. Adamidis IP, Spyropoulos MN. The effects of lymphadenoid hypertrophy on the position of the tongue, the mandible and the hyoid bone. Eur J Orthod 1983;5:287-94.

21. Schlenker WL, Jennings BD, Jeiroudi MT, Caruso JM. The effects of chronic absence of active nasal respiration on the growth of the skull: a pilot study. Am J Orthod Dentofac Orthop 2000;117:706-13.

22. Oulis CJ, Vadiakas GP, Ekonomides J, Dratsa J. The effect of hypertrophic adenoids and tonsils on the development of posterior crossbite and oral habits. J Clin Pediatr Dent. 1994;18:197-201.

23. Cheng MC, Enlow DH, Papsidero M, Broadbent BH Jr, Oyen O, Sabat M. Developmental effects of impaired breathing in the face of the growing child. Angle Orthod. 1988;58:309-20.

24. Linder-Aronson S, Woodside DG, Hellsing E, Emerson W. Normalization of incisor position after adenoidectomy. Am J Orthod. 1993;103:412 27.

25. Pereira FC, Motonaga SM, Faria PM, Matsumoto MA, Trawitzki LY, Lima SA. Myofunctional and cephalometric evaluation of mouth breathers. Rev Bras Otorrinolaringolol. 2001;67:43-9.

26. Lessa FC, Enoki C, Feres MF, Valera FC, Lima WT, Matsumoto MA. Breathing mode influence in craniofacial development. Braz Otorhinolaryngol. 2005;71:156-60.

27. Henrich DE, Hotson S, Drake AF, Warren DW. Monitoring nasal and oral airway patency. Cleft Palate Craniofac J. 1995;32:390-3.

28. McNamara JA. Influence of respiratory pattern on craniofacial growth. Angle Orthod. 1981;51:269-300.

29. Trotman CA, MCNamara JA Jr, Dibbets JM, van der Weele LT. Association of lip posture and the dimensions of the tonsils and sagitta airway with facial morphology. Angle Orthod. 1997;67:425-32.

30. Harvold EP, Tomer BS, Vargervik K, Chierici G. Primate experiments on oral respiration. Am J Orthod. 1981;79:359-72. 